VOL. $72(2005)$ [329-330]

\title{
On Singular Artin Monoids
}

\author{
Noelle Antony
}

This thesis is a combinatorial investigation of a class of monoids known as singular Artin monoids. The name of those monoids seems to suggest their origin. They include the singular braid monoid - a geometric extension of the braid group which exists in $\mathbb{C} \times[0,1]$ and was created for the sole purpose of studying Vassiliev invariants in knot theory. But they may also be construed (and indeed, are defined) as a formal extension of Artin groups which, in turn, naturally generalise braid groups. The thesis affords original discoveries within the literature and lends itself to alternative descriptions of certain preexisting results and problems. Each chapter contains an outline of its structure. Chapter 5 and part of Chapter 6 form the bulk of [2]; Chapter 2 together with the remainder of Chapter 6 will appear as [1]; Chapter 3 will be availabe as [3].

Chapter 1 establishes the necessary definitions and notation. After defining singular Artin monoids $\left(\mathcal{S} G_{M}\right)$, their "counter-parts", positive singular Artin monoids $\left(\mathcal{S} G_{M}^{+}\right)$, and their various types, we expound their place in the literature. More specifically, we discuss their relationship with other classes of monoids (which include Garside and chainable) and groups (Garside, Coxeter and Artin). We also state certain known facts and problems pertinent to the thesis.

In Chapter 2, we address the issue of divisibility in positive singular Artin monoids and review the relevant literature. The deductions we obtain are interesting in their own right, but they also have strong import in subsequent chapters.

Chapter 3 is devoted to showing that any $S G_{M}^{+}$naturally embeds into $\mathcal{S} G_{M}$.

In Chapter 4, the desingularisation map $(\eta)$ is defined, and (a generalised form of) "Birman's conjecture" is stated; namely, that $\eta$ injects from any singular Artin monoid into its corresponding group algebra. This is followed by a history of the discoveries regarding the conjecture. The inferences of Chapters 2 and 3 are then invoked to immediately acquire new results regarding the faithfulness of $\eta$.

In order to further investigate the aforementioned conjecture, a positive form is required. Chapter 5 sets up the requisite "machinery", and in Chapter 6 we infer that the restriction of $\eta$ to certain submonoids of $\mathcal{S} G_{M}$ is faithful, expanding on the deductions

Received 2nd August, 2005

Thesis submitted to The University of Sydney, November 2004. Degree approved, May 2005. Supervisor: Dr. David Easdown. Associate Supervisor: Dr. Andrew Mathas.

Copyright Clearance Centre, Inc. Serial-fee code: 0004-9727/05 \$A2.00+0.00. 
made in Chapter 4. We conclude the thesis in Chapter 7 by drawing on all of our results to prove that the conjecture is equivalent to another (more easily manipulated) statement.

\section{REFERENCES}

[1] N. Antony, 'On singular Artin monoids and contributions to Birman's conjecture', Comm. Algebra (to appear).

[2] N. Antony, 'On the injectivity of the Vassiliev homomorphism of singular Artin monoids', Bull. Austral. Math. Soc. 70 (2004), 401-422.

[3] N. Antony, 'The natural embedding of positive singular Artin monoids', Comm. Algebra (to appear).

School of Mathematics and Statistics

The University of Sydney

New South Wales 2006

Australia 